\title{
Betulinic acid inhibits autophagic flux and induces apoptosis in human multiple myeloma cells in vitro
}

\author{
Li-jing YANG", Yan CHEN", Jing HE, Sha YI, Lu WEN, Jie ZHAO, Ben-ping ZHANG, Guo-hui CUI* \\ Department of Hematology, Union Hospital, Tongji Medical College, Huazhong University of Science and Technology, Wuhan 430022, \\ China
}

\begin{abstract}
Aim: To investigate the effects of betulinic acid (BA) on apoptosis and autophagic flux in multiple myeloma cells and the relationship between the two processes.

Methods: The proliferation of human multiple myeloma KM3 cells was measured with MTT assay. FITC/PI double-labeled flow cytometry (FCM) and Hoechst 33258 staining were used to analyze the cell apoptosis. Caspase 3, PARP, Beclin1, LC3-II, and P62 were detected using Western blotting.

Results: Treatment of KM3 cells with BA $(5-25 \mu \mathrm{g} / \mathrm{mL})$ suppressed the cell proliferation in time- and dose-dependent manners. The $\mathrm{IC}_{50}$ values at 12,24 , and $36 \mathrm{~h}$ were $22.29,17.36$, and $13.06 \mu \mathrm{g} / \mathrm{mL}$, respectively. BA treatment dose-dependently induced apoptosis of KM3 cells, which was associated with the activation of caspase 3. However, Z-DEVD-FMK, a specific inhibitor of caspase 3, did not decrease, but rather sensitized the cells to BA-induced apoptosis, suggesting an alternative mechanism involved. On other hand, BA treatment dose-dependently increased the accumulation of LC3-II and P62 in KM3 cells, representing the inhibition of autophagic flux. Furthermore, BA treatment dose-dependently downregulated the expression of Beclin 1, an important inducer of autophagy, in KM3 cells. In the presence of BA, Z-DEVD-FMK induced autophagy and increased the amount of LC3-II in KM3 cells, which may occur via attenuating BA-induced decrease in the level of Beclin 1. Similarly, rapamycin, an autophagy inducer, increased the amount of LC3-II in KM3 cells. In the presence of BA, rapamycin caused further increase in the amount of LC3-II. Furthermore, rapamycin sensitized BA-treated KM3 cells to apoptosis.

Conclusion: The results demonstrate that BA induces apoptosis and blocks autophagic flux in KM3 cells. Furthermore, in addition to activation of caspase 3 , the inhibition of autophagic flux also contributes to the BA-mediated apoptosis of KM3 cells.
\end{abstract}

Keywords: multiple myeloma; betulinic acid; Z-DEVD-FMK; rapamycin; apoptosis; caspase 3; autophagy; autophagic flux; Beclin 1; LC3- II

Acta Pharmacologica Sinica (2012) 33: 1542-1548; doi: 10.1038/aps.2012.102; published online 15 Oct 2012

\section{Introduction}

Multiple myeloma (MM), as a clonal plasma malignancy, is characterized by osteolytic bone lesions, the production of monoclonal proteins, renal disease, immunodeficiency, anemia and hypercalcemia ${ }^{[1]}$. Historically, the prognosis of MM patients has been very poor owing to the lack of effective therapy, although the introduction of lenalidomide and bortezomib (a proteasome inhibitor) has dramatically improved the outcome of MM patients in recent decades. However, MM remains incurable, and almost all patients will experience a relapse at some point in their lifetime ${ }^{[2]}$. Therefore, it is urgent to identify new therapeutic targets and to exploit additional

\footnotetext{
"These authors contributed equally to this paper.

* To whom correspondence should be addressed.

E-mail ghcui@medmail.com.cn

Received 2012-05-20 Accepted 2012-07-01
}

anti-MM agents that are more potent but less toxic.

Excess immunoglobulin (Ig) is synthesized in MM, generating a significant amount of endoplasmic reticulum (ER)localized, unfolded or misfolded protein that is potentially toxic to $\mathrm{MM}$ cells ${ }^{[3]}$. For this reason, autophagy may play an important role in maintaining the stability of the intracellular environment and in the resistance to cell death of MM cells. Autophagy is a catabolic membrane-trafficking process that is mainly involved in the sequestration and degradation of long-lived intracellular proteins and organelles ${ }^{[4]}$. During autophagy, the cytoplasm and organelles are sequestered into double membrane-bound vesicles (autophagosomes), and the autophagosomes are then delivered to the lysosomes by a fusion process, leading to the generation of autolysosomes in which the cargo is hydrolyzed ${ }^{[5]}$. The completion of autophagic flux eliminates all of the damaged cellular constituents and provides catabolic intermediates to the cell when 
the exogenous energy supplies are insufficient ${ }^{[5]}$. In fact, the level of autophagy in MM cells is significantly higher than that in normal plasma cells. It has been demonstrated that inhibiting autophagy by Beclin 1-siRNA or chloroquine results in MM cell death ${ }^{[3]}$. Furthermore, autophagy protects MM cells against apoptosis under nutrient depletion conditions ${ }^{[6]}$ and during drug resistance ${ }^{[7]}$. These accumulating pieces of evidence indicate that autophagy could be a promising new target for anti-MM therapy.

Betulinic acid (BA), a natural pentacyclic triterpene isolated from birch, exhibits a variety of biological activities, including anti-HIV, anti-HBV, anti-malarial ${ }^{[8-10]}$ and potent anti-tumor properties in melanoma ${ }^{[11]}$, leukemia ${ }^{[12]}, \operatorname{colon}^{[13]}, \operatorname{lung}^{[14]}$, gliomas $^{[15]}$, prostate carcinoma ${ }^{[16]}$, gastric adenocarcinoma ${ }^{[17]}$ and multiple myeloma ${ }^{[18,19]}$. Various mechanisms have been reported for the extensive anticancer activity of BA, including directly triggering mitochondrial membrane permeabilization $^{[20]}$, regulating Bcl-2 family proteins ${ }^{[21]}$, downregulating Hiwi expression ${ }^{[17]}$ and suppressing the activation of the STAT3 pathway through the induction of the protein tyrosine phosphatase SHP-1 ${ }^{[19]}$. However, there are no reports as to whether BA has any effects on cancer cell autophagy.

In this study, we investigated the efficiency of BA with regard to apoptosis and autophagy in multiple myeloma KM3 cells and explored the relationship between these processes. We demonstrated that BA exhibited a potent inhibition of KM3 cell proliferation, induction of apoptosis and inhibition of autophagic flux. Moreover, the autophagic flux inhibition contributed to the BA-induced apoptosis of the KM3 cells.

\section{Materials and methods}

\section{Reagents and cell culture}

BA $\left(\mathrm{C}_{30} \mathrm{H}_{48} \mathrm{O}_{3}\right.$, molecular weight $\left.=456.7\right)$, rapamycin, dimethyl sulfoxide (DMSO), Hoechst 33258 and 3-(4,5-dimethyl-2thiazolyl)-2,5-diphenyl-2H-tetrazolium (MTT) were purchased from Sigma-Aldrich, USA. Z-DEVD-FMK was purchased from Medical and Biological Laboratories Co, Ltd, Japan. The Annexin V-fluorescein isothiocyanate (FITC)/propidium iodide (PI) reagent kit was purchased from Nanjing Key-Gen Biotech Co, Ltd, China. The BCA Protein Assay kit, chemiluminescence reagent kit and PVDF membranes were purchased from Pierce Biotechnology, Inc, USA. The anti-caspase 3, anti-PARP, anti-Beclin 1 and anti- - -tubulin antibodies were provided by Cell Signaling Technology, Inc, USA. The antiLC3 and anti-P62 antibodies were purchased from Abcam, USA, and Santa Cruz Biotechnology, Inc, USA, respectively. The anti-rabbit and anti-mouse secondary antibodies were purchased from Jackson Immuno Research Laboratories, Inc, USA. To describe the methodology briefly, BA was dissolved in DMSO, aliquoted, stored at $-20^{\circ} \mathrm{C}$ and thawed prior to use. The RPMI-1640 medium and fetal bovine serum (FBS) were obtained from Gibco Co, USA, and Hangzhou Sijiqing Biological Engineering Materials Co, Ltd, China, respectively. The human multiple myeloma KM3 cells were obtained from the Department of Immunology, Tongji Medical College, Huazhong University of Science and Technology, China, and were maintained in RPMI-1640 medium supplemented with 10\% FBS in a $5 \% \mathrm{CO}_{2}$ humidified atmosphere at $37^{\circ} \mathrm{C}$.

\section{MTT assay}

The effects of BA on the proliferation of KM3 cells were detected using the MTT assay. Briefly, KM3 cells were maintained in RPMI-8226 medium until the mid-log phase. The cells were then seeded in 96-well plates with or without BA at various concentrations $(5,10,15,20$, and $25 \mu \mathrm{g} / \mathrm{mL})$ at a density of $2 \times 10^{4}$ cells per well. After incubation for a set period of time, $20 \mu \mathrm{L}$ MTT ( $5 \mathrm{mg} / \mathrm{mL}$ ) was added, and the cells were incubated for another $3 \mathrm{~h}$ at $37^{\circ} \mathrm{C}$. The supernatant was discarded, and $150 \mu \mathrm{L}$ DMSO was added. The plate was gently agitated until the blue formazan crystals were fully dissolved. The absorbance $(A)$ was measured at $490 \mathrm{~nm}$ using a plate microreader (Tecan Spectra, Switzerland), and the cell proliferation inhibition ratio (\%) was calculated using the following formula: [1-( $A$ of experimental sample/ $A$ of the control sample) $] \times 100$.

\section{Annexin V-FITC/PI double-labeled flow cytometry}

The apoptosis ratio was measured in KM3 cells treated with BA alone or in combination with Z-DEVD-FMK or rapamycin. Two-color flow cytometry (FCM) was applied to detect the expression of Annexin V-FITC and the exclusion of PI. The cells positive for Annexin V-FITC and negative for PI represented the early apoptotic cells, whereas the cells positive for both markers represented the late apoptotic cells. The total apoptosis ratio was the sum of the early and late apoptotic cells. Briefly, KM3 cells were collected after the treatment using EP tubes, washed twice with PBS and resuspended in $500 \mu \mathrm{L}$ binding buffer. A total of $5 \mu \mathrm{L}$ of Annexin V-FITC was added, and the samples were maintained at room temperature for $10 \mathrm{~min}$. Next, $5 \mu \mathrm{L}$ of PI was added, and the cells were incubated for another $10 \mathrm{~min}$ in the dark. The fluorescence intensity was detected using a flow cytometer (Becton-Dickinson, Franklin Lakes, NJ, USA).

\section{Hoechst 33258 staining}

KM3 cells were treated with $20 \mu \mathrm{g} / \mathrm{mL}$ BA for $24 \mathrm{~h}$, and the nuclear fragmentation was visualized using Hoechst 33258 staining. Briefly, $1 \times 10^{5}$ cells were seeded in 12 -well plates and incubated with BA. After $24 \mathrm{~h}$, the cells were collected, washed twice with PBS and fixed in $4 \%$ paraformaldehyde for $10 \mathrm{~min}$ at room temperature before being deposited on polylysine-coated slides. After $30 \mathrm{~min}$, the adhered cells were permeabilized by incubation with $0.1 \%$ Triton $X-100$ for 5 min at $4{ }^{\circ} \mathrm{C}$. The cells were then incubated with Hoechst 33258 for $30 \mathrm{~min}$ at room temperature, rinsed with PBS and mounted on coverslips using glycerol. Lastly, the images of the nuclear changes were visualized using an Olympus BH-2 fluorescence microscope (Tokyo, Japan).

\section{Western blot analysis}

All of the KM3 cells treated with BA alone or in combination with Z-DEVD-FMK or rapamycin for $24 \mathrm{~h}$ were collected. The 
cells were washed twice with PBS and completely lysed in a lysis buffer containing protease inhibitors. The extracts were centrifuged at $12000 \times \mathrm{g}$ for $15 \mathrm{~min}$ at $4^{\circ} \mathrm{C}$, and the clear supernatants containing the total protein were isolated. The protein concentration was quantified using the BCA assay. SDS-polyacrylamide gel electrophoresis ( $40 \mu \mathrm{g}$ of protein per lane) was performed, and the proteins were then transferred to PVDF membranes. The membranes were blocked in 5\% non-fat milk for $2 \mathrm{~h}$ at room temperature and then probed with the specific primary antibody and corresponding secondary antibody. The specific protein bands were visualized using an ECL kit.

\section{Statistical analysis}

Each experiment was repeated three times. The data are presented as the mean \pm SD and analyzed using SPSS 13.0 statistical software for Windows (USA). The comparisons between each group were analyzed using a one-way ANOVA and Student-Newman-Keuls (SNK) test; $P<0.05$ was considered statistically significant.

\section{Results}

\section{BA treatment inhibited the proliferation of KM3 cells}

The MTT assay was employed to detect the cytotoxicity of different concentrations of BA $(0,5,10,15,20$, and $25 \mu \mathrm{g} / \mathrm{mL})$ on KM3 cells for 12,24 , and $36 \mathrm{~h}$. The results demonstrated that BA treatment significantly decreased KM3 cell proliferation in a time- and dose-dependent manner. The $\mathrm{IC}_{50}$ values for BA in $\mathrm{KM} 3$ cells at 12,24 , and $36 \mathrm{~h}$ were $22.29,17.36$, and 13.06 $\mu \mathrm{g} / \mathrm{mL}$, respectively (Figure 1 ).

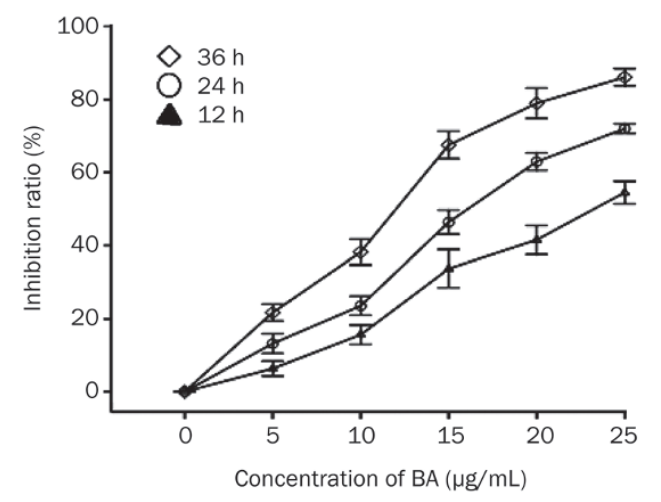

Figure 1. Assessment of the BA-induced proliferation inhibition ratio in $\mathrm{KM} 3$ cells. The cells were treated with various concentrations of BA for 12 , 24 , and $24 \mathrm{~h}$. MTT assay was applied to detect the proliferation inhibition ratio. Data were reported as the mean $\pm S D$ of three independent experiments.

\section{BA treatment induced apoptosis in KM3 cells}

Annexin V-FITC/PI double-labeled flow cytometry was utilized to assess the apoptosis ratio of KM3 cells treated with BA. Whereas the early apoptotic cells were Annexin V-FITC positive and PI negative, the late apoptotic cells were both
Annexin V-FITC and PI positive. The total apoptosis ratio was the sum of the early apoptosis and late apoptosis ratios. The apoptosis rates for KM3 cells treated with 10, 15, and 20 $\mathrm{\mu g} / \mathrm{mL}$ BA for $24 \mathrm{~h}$ were $15.60 \% \pm 1.58 \%, 23.86 \% \pm 2.06 \%$, and $44.12 \% \pm 3.22 \%$, respectively, which was significantly higher than that of the control group, at $4.74 \% \pm 0.82 \%$ (Figure 2).

KM3 cells treated with BA for $24 \mathrm{~h}$ were stained with Hoechst 33258 to visualize the nuclear changes. KM3 cell nuclei were regular in shape in the control group, whereas apparent apoptotic bodies were observed in KM3 cells after treatment with $20 \mu \mathrm{g} / \mathrm{mL}$ BA. The nuclei in these apoptotic cells were condensed, and the nuclear envelopes appeared lytic (Figure 3).

\section{BA-induced apoptosis in KM3 cells was mediated through caspase 3 activation}

Western blotting was used to explore the role of caspase 3 in the BA-induced apoptosis of KM3 cells. The level of caspase 3 activation and the cleavage of PARP (the substrate of activated caspase 3) were evaluated. The results showed that BA treatment had a dose-dependent effect on the activation of caspase 3; consistent with the increase in activated caspase 3, and the level of cleaved PARP also increased (Figure 4). These results demonstrated that caspase 3 activation was involved in the progression of BA-induced apoptosis in KM3 cells; however, other mechanisms might be contributing as well.

\section{Z-DEVD-FMK blocked the BA-induced caspase 3 activation but} sensitized the BA-treated KM3 cells for apoptosis

To further identify whether caspase 3 is the only crucial factor for the apoptosis of KM3 cells, a specific caspase 3 inhibitor (Z-DEVD-FMK) was used in this experiment. Unlike BA, Z-DEVD-FMK significantly blocked the cleavage of PARP (Figure 5B), indicating that Z-DEVD-FMK relieved the BAinduced activation of caspase 3. Surprisingly, Z-DEVD-FMK did not decrease the apoptosis ratio of BA-treated KM3 cells but, rather, sensitized them for apoptosis (Figure 2).

\section{BA treatment of KM3 cells inhibited autophagic flux}

To detect the autophagic flux, LC3 conversion and P62 expression were assessed using Western blotting. Based on autophagy detection guidelines, an increase in LC3 conversion but not in P62 expression represents the upregulation of autophagic flux, whereas an increase in LC3 conversion and P62 expression represents the inhibition of autophagic flux ${ }^{[22]}$. In our experiment, BA treatment increased the expression of LC3-II and P62 in a dose-dependent manner (Figure 6). These results demonstrated that the increase in the LC3-II protein, which represented the number of autophagosomes, was not due to the induction of autophagy but instead to the inhibition of the autophagic flux.

BA treatment downregulated Beclin 1 via caspase 3 activation, whereas Z-DEVD-FMK alleviated the Beclin 1 downregulation by blocking caspase 3 activation and inducing autophagy

It has previously been reported that activated caspase 3 is 

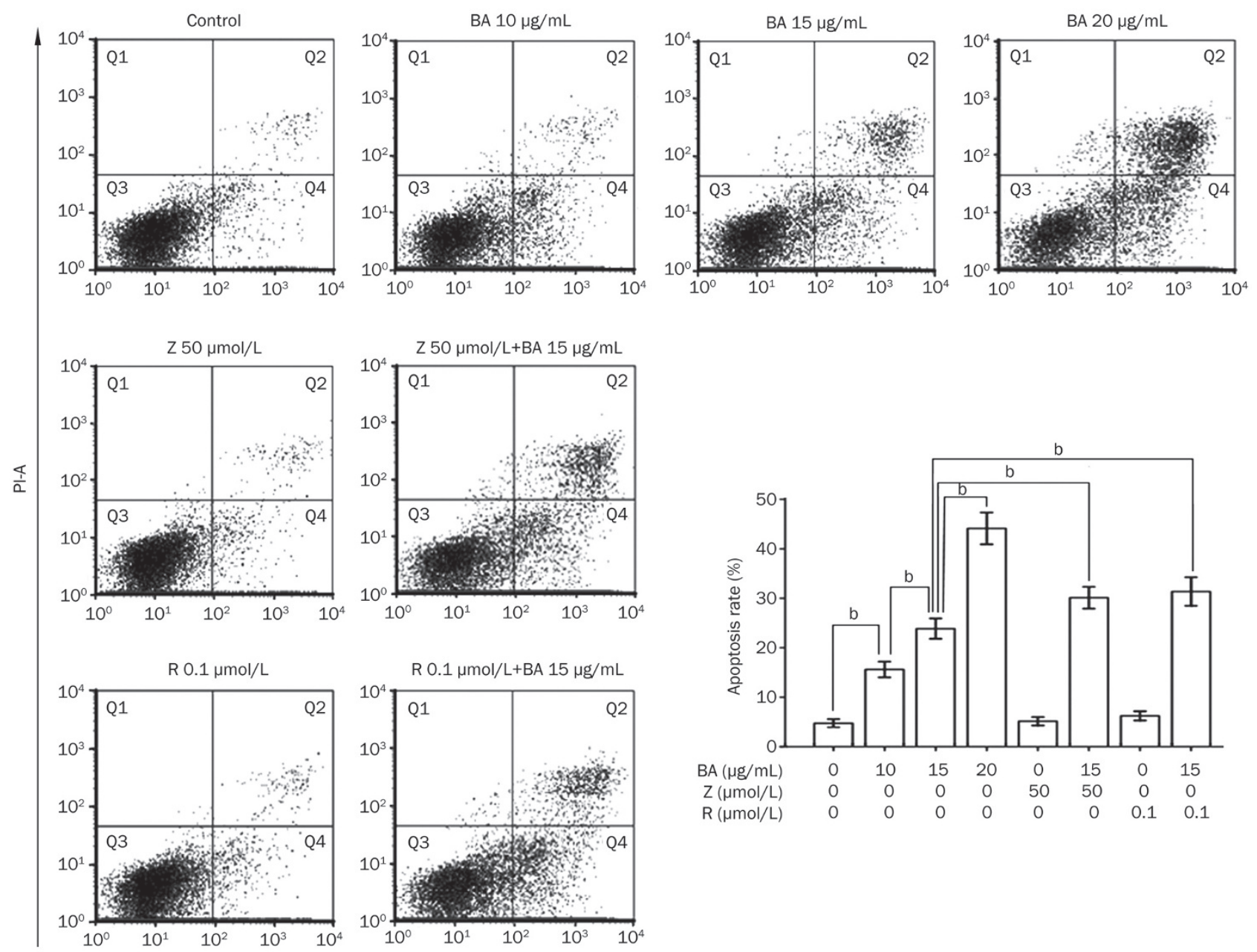

FITC-A

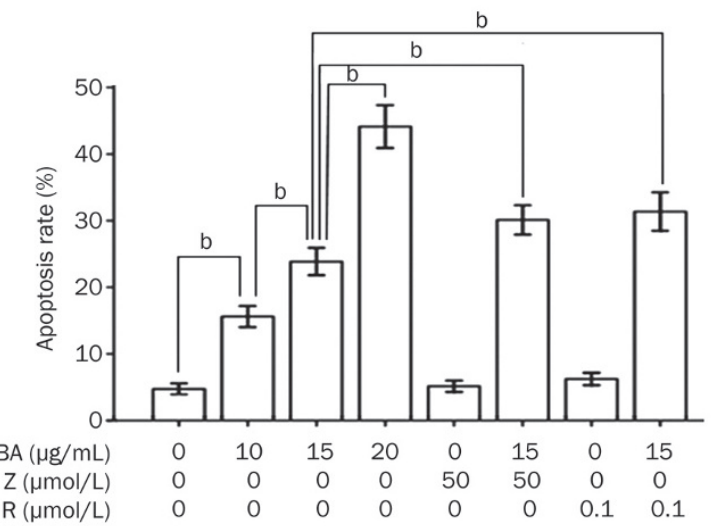

Figure 2. Assessment of apoptosis rate in KM3 cells treated with BA for $24 \mathrm{~h}$ in the absence or presence of Z-DEVD-FMK or rapamycin. Annexin V-FITC/ PI double staining was applied to measure apoptosis ratio in KM3 cells using FCM. Q2 quadrant and Q4 quadrant represented late and early apoptotic cells, respectively. The total apoptosis rate was the sum of these two. Data were reported as the mean $\pm S D$ of three independent experiments. ${ }^{\mathrm{b}} \mathrm{P}<0.05$. BA, betulinic acid; Z, Z-DEVD-FMK; R, rapamycin.
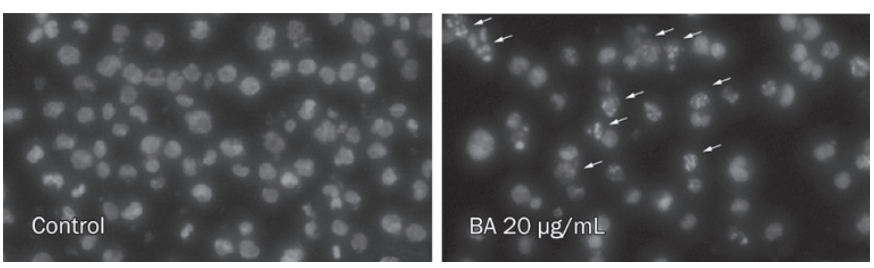

Figure 3. The effect of $\mathrm{BA}$ on the apoptosis of $\mathrm{KM} 3$ cells. After being incubated with $20 \mu \mathrm{g} / \mathrm{mL}$ of $B A$ for $24 \mathrm{~h}$, the cells were stained with Hoechst 33258 and visualized using a fluorescence microscope. A number of apoptotic bodies exhibiting fragmented or condensed nuclei (indicated by the arrows) were detected in the BA-treated group but not in the control group.

able to cleave Beclin 1, which is an important inducer of autophagy ${ }^{[23]}$. Accordingly, we confirmed that BA downregulated the expression of Beclin 1 in a dose-dependent manner

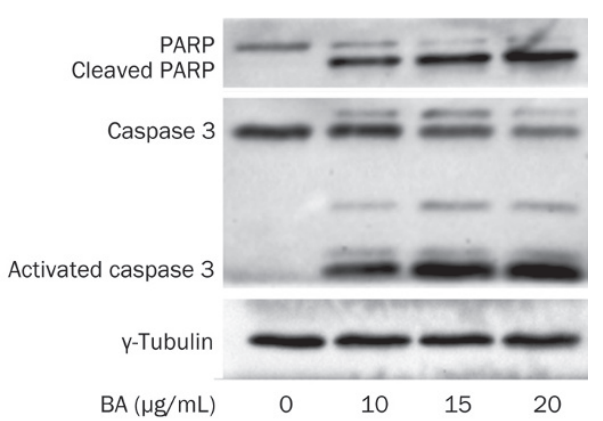

Figure 4. The effect of BA on caspase 3 and PARP. KM3 cells were incubated with indicated concentrations of BA for $24 \mathrm{~h}$. Western blot analysis was applied to analyze the caspase 3 activation and PARP cleavage (a major substrate of activated caspase 3 ). As shown in the figure, BA treatment activated caspase 3 accompanied with the PARP cleavage in a dose-dependent manner. The figures are representative of three separate experiments. 
A
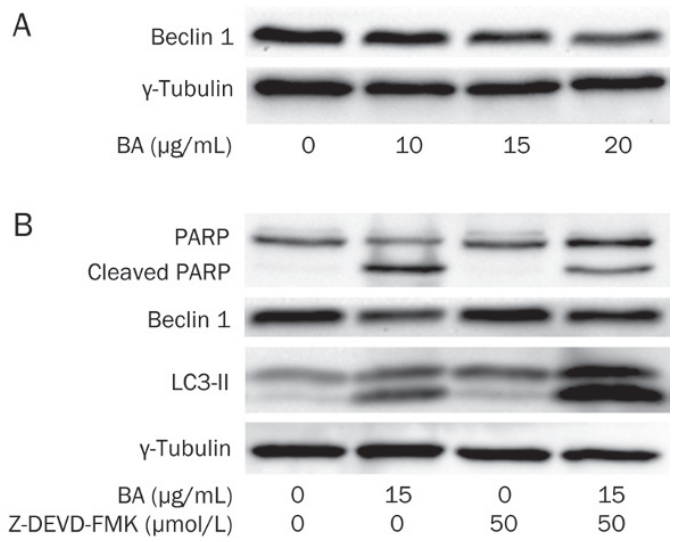

Figure 5. The effect of Z-DEVD-FMK on KM3 cells in the presence or absence of BA. Panel A: BA downregulated the levels of Beclin 1 in KM3 cells in a dose-dependent manner. Panel B: Z-DEVD-FMK alone did not significantly affect PARP, Beclin 1 and LC3-II but obviously decreased the cleavage of PARP, the decrease of Beclin 1 in KM3 cells caused by BA. Furthermore, Z-DEVD-FMK further increased the level of LC3-II in KM3 cells in the presence of BA. This confirmed that Z-DEVD-FMK induced autophagy when combined with BA. The figures are representative of three separate experiments.

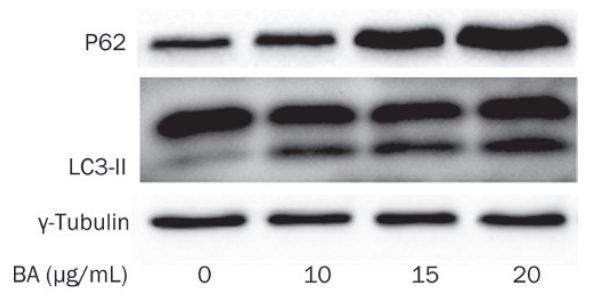

Figure 6. The effect of BA on autophagic flux in KM3 cells. KM3 cells were treated with BA and LC3-II and P62 were applied to analyze the autophagic flux using Western blot analysis. The results showed that BA increased the amount of LC3-II and P62 in a dose-dependent manner, which indicated that BA inhibited the autophagic flux in KM3 cells. The figures are representative of three separate experiments.

(Figure 5A), a result that was accompanied by the activation of Caspase 3 and PARP cleavage (Figure 4). Although Z-DEVDFMK alone was unable to alter the level of Beclin 1, Z-DEVDFMK could significantly alleviate the decrease of Beclin 1 induced by BA. As a result, the LC3-II level was increased even higher in the KM3 cells treated with BA combined with Z-DEVD-FMK compared to BA alone (Figure 5B). These results indicated that, when combined with BA, Z-DEVD-FMK had the potential to induce KM3 cell autophagy mediated by Beclin 1.

Rapamycin induced autophagy in KM3 cells and sensitized them to apoptosis when exposed to BA

It has been reported that the inhibition of autophagic flux by chloroquine contributes to cell death ${ }^{[24]}$. However, there has been no report on whether BA could inhibit autophagic flux in multiple myeloma cells or on the relationship between apoptosis and autophagic flux inhibition in myeloma cells. Therefore, we used the autophagy inducer rapamycin in this study. As expected, rapamycin alone increased the expression of LC3-II (Figure 7), which indicated the induction of autophagy without any effects on the apoptotic rate (Figure 2) or the activation of caspase 3 (Figure 7) in KM3 cells. BA treatment in the presence of rapamycin for $24 \mathrm{~h}$ increased the amount of LC3-II when compared to the level of either of these reagents alone (Figure 7). Furthermore, rapamycin sensitized BA-treated KM3 cells to apoptosis: the treatment with both BA and rapamycin contributed to apoptosis in $31.37 \% \pm 2.88 \%$ of KM3 cells, which was significantly higher than that of treatment with BA alone (Figure 2). Moreover, the treatment with both $\mathrm{BA}$ and rapamycin resulted in more cleavage of caspase 3 and PARP compared to treatment with BA alone. These results implied that the blockage of the autophagic flux might mediate the apoptotic progress in BA-treated KM3 cells. In this particular situation, inducing autophagy using rapamycin or even the caspase 3 inhibitor Z-DEVD-FMK would strengthen the potential of BA to induce KM3 cell apoptosis.

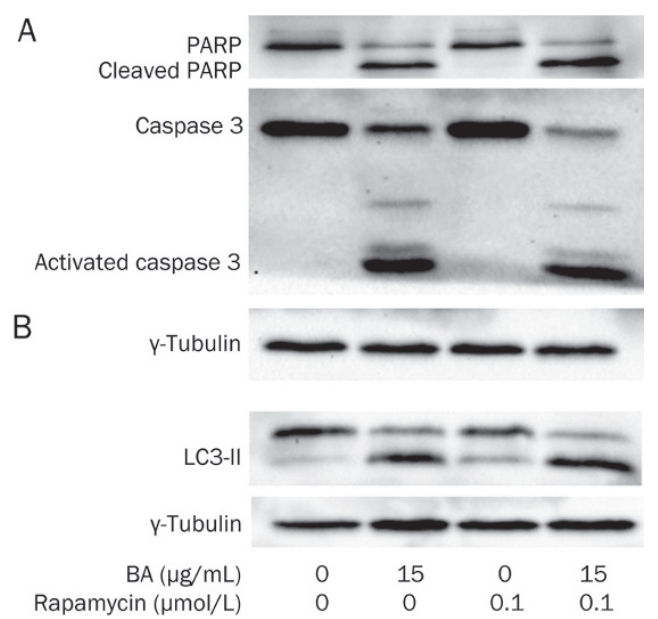

Figure 7. The effect of rapamycin on KM3 cells in the presence or absence of BA. Panel A: Rapamycin alone had no effect on caspase 3 and PARP in KM3 cells but sensitized the KM3 cells for the BA-induced cleavage of caspase 3 and PARP. Panel B: Rapamycin induced the autophagy in KM3 cells. When rapamycin was combined with BA, it led to further accumulation of LC3-II in KM3 cells as compared to that of the group treated with $\mathrm{BA}$ alone. The figures are representative of three separate experiments.

\section{Discussion}

BA, a naturally occurring pentacyclic triterpene, has potent anti-tumor properties toward tumors of various origins, both in vitro and in vivo. Interestingly, BA has been reported to be less toxic to cells from healthy tissues than to cancer cells. For example, normal melanocytes remained relatively resistant to BA, compared to melanoma cells, as measured by prolifera- 
tion and apoptosis analyses ${ }^{[25]}$. Peripheral blood lymphocytes and human skin fibroblasts were also identified as being highly resistant to BA treatment ${ }^{[26]}$. Moreover, a complete lack of toxicity was observed at a concentration up to $100 \mathrm{mg} / \mathrm{kg}$ body weight in animal studies ${ }^{[26]}$. These characteristics of BA highlight it as a potential anticancer agent.

Multiple mechanisms have been reported for the potent anticancer activity of $\mathrm{BA}^{[21]}$. In a previous study, we demonstrated that BA induced cell cycle arrest and apoptosis in human gastric adenocarcinoma AGS cells through the downregulation of Hiwi and Cyclin $\mathrm{B}^{[17]}$. In the present study, BA inhibited cell proliferation in multiple myeloma KM3 cells and induced apoptosis in a dose-dependent manner. The $\mathrm{IC}_{50}$ values for BA in KM3 cells at 12, 24, and $36 \mathrm{~h}$ were 22.29, 17.36, and $13.06 \mu \mathrm{g} / \mathrm{mL}$, respectively (Figure 1). It has been reported that the activation of caspases contributed to the BAinduced apoptosis in neuroectodermal tumors ${ }^{[27]}$. Similarly, we found that caspase 3 cleavage had a role in the progression of the BA-induced apoptosis in multiple myeloma KM3 cells. The treatment of KM3 cells with various concentrations of $\mathrm{BA}(10,15$, and $20 \mu \mathrm{g} / \mathrm{mL})$ resulted in apoptosis rates of $15.60 \% \pm 1.58 \%, 23.86 \% \pm 2.06 \%$, and $44.12 \% \pm 3.22 \%$, respectively, that were significantly higher than that of the control group (Figure 2). BA also contributed to caspase 3 activation in a dose-dependent manner, followed by the cleavage of PARP (Figure 4), a factor that is considered one of the major cleavage targets of caspase 3 and is involved in DNA repair in response to extracellular stress ${ }^{[28]}$. These results demonstrated that the activation of caspase 3 mediated the process of the observed BA-induced apoptosis in KM3 cells.

To identify whether caspase 3 was the only key factor mediating apoptosis in KM3 cells, a special caspase 3 inhibitor, Z-DEVD-FMK, was employed in this experiment. Surprisingly, Z-DEVD-FMK enhanced the BA-induced apoptosis in KM3 cells (Figure 2), even though the caspase 3 pathway was inhibited by Z-DEVD-FMK (Figure 5). This result indicated that there might be another mechanism through which BA exerted toxicity in KM3 cells.

Accumulating evidence has demonstrated that autophagy favors the survival of MM cells ${ }^{[3,6]}$. Therefore, we hypothesize that changes in autophagy may be involved in the mechanism of BA-induced apoptosis in KM3 cells. The results of this study demonstrated that BA treatment significantly increased the concentration of the LC3-II protein in KM3 cells in a dosedependent manner. However, the accumulation of LC3-II, which reflects the induction of autophagy and/or inhibition of autophagosome clearance, is insufficient to measure the autophagic flux. The autophagic flux refers to the complete process of autophagy, including the delivery of the cargo to the lysosomes via the fusion of the latter with the autophagosomes and its subsequent breakdown and recycling. Furthermore, we detected changes in the concentration of $\mathrm{P}_{62}{ }^{[22]}$ in KM3 cells treated with various concentrations of BA. A 24-h BA treatment of KM3 cells upregulated the level of P62 in a dose-dependent manner, similar to the changes in the LC3-II concentration. According to the guidelines ${ }^{[22,29]}$, we can con- clude that BA can block the autophagic flux in KM3 cells in a dose-dependent manner.

It has been reported that caspase 3 inhibited autophagy in its early stages by cleaving the autophagy inducer Beclin 1 at its $149 \mathrm{D}$ site $^{[23]}$. We also found that the gradual activation of caspase 3 was accompanied by the downregulation of Beclin 1 in KM3 cells, whereas the BA concentration gradually increased (Figure 5). Moreover, Z-DEVD-FMK reversed the BA-induced Beclin 1 downregulation by blocking caspase 3 (Figure 5). Although Z-DEVD-FMK alone did not induce autophagy, when it was combined with BA, the upregulation of LC3-II became more pronounced in comparison to that of the BA-treated group (Figure 5). This finding was an indication that more autophagosomes had accumulated and were not digested by the lysosomes for recycling, demonstrating that Z-DEVD-FMK induced autophagy in KM3 cells by inhibiting the down-regulation of Beclin 1 in the presence of BA. This might explain the increased apoptosis rate in the KM3 cells in spite of the reduction in the caspase 3 activity by Z-DEVD-FMK. In this situation, more proteins and organelles are sequestered by the autophagosomes, however the cargo cannot be recycled to provide catabolic intermediates to sustain the cells under stress.

To further verify that autophagic flux inhibition mediates the BA-induced apoptosis in KM3 cells, we applied a recognized inducer of autophagy, rapamycin, to these cells. The results showed that rapamycin alone was able to induce autophagy in KM3 cells and did not have toxic effects. However, when rapamycin was added in combination with BA, the apoptosis rate and the concentration of LC3-II were significantly increased in the KM3 cells (Figure 2 and Figure 7, respectively). These results further confirmed that autophagic flux inhibition contributed to the observed BA-induced apoptosis in KM3 cells in spite of alleviating the activity of caspase 3.

Furthermore, the combination of rapamycin and BA contributed to a higher degree of cleavage for caspase 3 and PARP compared to that of BA alone. We concluded that the further inhibition of autophagic flux led to the further activation of caspase 3 and cleavage of PARP. Although Z-DEVD-FMK inhibited caspase 3, the rate of BA-induced apoptosis was not reduced in KM3 cells, showing that the inhibition of the autophagic flux must have triggered other pathways that contributed to apoptosis.

In summary, these results demonstrated that BA acts as a potent growth inhibitor and apoptosis inducer in KM3 cells. Furthermore, we demonstrated that BA inhibited autophagic flux, which contributed to apoptosis to a certain extent. As autophagy plays an important role in cell survival during stress and drug resistance ${ }^{[30,31]}$, our results indicated that BA can both kill cancer cells and can also reduce the incidence of chemotherapy resistance if used for cancer therapy.

\section{Acknowledgements}

This work was supported by the National Natural Science Foundation of China (№ 30871036 and № 81070429$)$. 


\section{Author contribution}

Yan CHEN, Huo-hui CUI, and Li-jing YANG designed research; Li-jing YANG performed research; Jing HE, Sha YI, Lu WEN, Jie ZHAO, and Ben-ping ZHANG managed analytical tools and reagents; Li-jing YANG analyzed data and wrote the paper.

\section{References}

1 Podar K, Tai YT, Hideshima T, Vallet S, Richardson PG, Anderson KC. Emerging therapies for multiple myeloma. Expert Opin Emerg Drugs 2009; 14: 99-127.

2 Siegel DS, Vij R, Jakubowiak AJ. Clinical roundtable monograph. Emerging treatment options for relapsed and refractory multiple myeloma. Clin Adv Hematol Oncol 2011; 9: 1-15.

3 Hoang B, Benavides A, Shi Y, Frost P, Lichtenstein A. Effect of autophagy on multiple myeloma cell viability. Mol Cancer Ther 2009; 8: 1974-84.

4 Mizushima N, Levine B, Cuervo AM, Klionsky DJ. Autophagy fights disease through cellular self-digestion. Nature 2008; 451: 1069-75.

5 Tschan MP, Simon HU. The role of autophagy in anticancer therapy: promises and uncertainties. J Intern Med 2010; 268: 410-8.

6 Zeng R, He J, Peng J, Chen Y, Yi S, Zhao F, et al. The time-dependent autophagy protects against apoptosis with possible involvement of Sirt1 protein in multiple myeloma under nutrient depletion. Ann Hematol 2012; 91: 407-17.

7 Zeng R, Chen Y, Zhao S, Cui GH. Autophagy counteracts apoptosis in human multiple myeloma cells exposed to oridonin in vitro via regulating intracellular ROS and SIRT1. Acta Pharmacol Sin 2012; 33: $91-100$.

8 Theo A, Masebe T, Suzuki Y, Kikuchi H, Wada S, Obi CL, et al. Peltophorum africanum, a traditional South African medicinal plant, contains an anti HIV-1 constituent, betulinic acid. Tohoku J Exp Med 2009; 217: 93-9.

9 Yao D, Li H, Gou Y, Zhang H, Vlessidis AG, Zhou H, et al. Betulinic acid-mediated inhibitory effect on hepatitis $B$ virus by suppression of manganese superoxide dismutase expression. FEBS J 2009; 276: 2599-614.

10 Ziegler HL, Franzyk H, Sairafianpour M, Tabatabai M, Tehrani MD, Bagherzadeh $\mathrm{K}$, et al. Erythrocyte membrane modifying agents and the inhibition of Plasmodium falciparum growth: structure-activity relationships for betulinic acid analogues. Bioorg Med Chem 2004; 12: 119-27.

11 Pisha E, Chai H, Lee IS, Chagwedera TE, Farnsworth NR, Cordell GA, et al. Discovery of betulinic acid as a selective inhibitor of human melanoma that functions by induction of apoptosis. Nat Med 1995; 1 : 1046-51.

12 Ehrhardt H, Fulda S, Fuhrer M, Debatin KM, Jeremias I. Betulinic acidinduced apoptosis in leukemia cells. Leukemia 2004; 18: 1406-12.

13 Jung GR, Kim KJ, Choi CH, Lee TB, Han SI, Han HK, et al. Effect of betulinic acid on anticancer drug-resistant colon cancer cells. Basic Clin Pharmacol Toxicol 2007; 101: 277-85.

14 Mullauer FB, van Bloois L, Daalhuisen JB, Ten Brink MS, Storm G, Medema JP, et al. Betulinic acid delivered in liposomes reduces growth of human lung and colon cancers in mice without causing sys- temic toxicity. Anticancer Drugs 2011; 22: 223-33.

15 Rzeski W, Stepulak A, Szymanski M, Sifringer M, Kaczor J, Wejksza $\mathrm{K}$, et al. Betulinic acid decreases expression of bcl-2 and cyclin D1, inhibits proliferation, migration and induces apoptosis in cancer cells. Naunyn Schmiedebergs Arch Pharmacol 2006; 374: 11-20.

16 Chintharlapalli S, Papineni S, Ramaiah SK, Safe S. Betulinic acid inhibits prostate cancer growth through inhibition of specificity protein transcription factors. Cancer Res 2007; 67: 2816-23.

17 Yang LJ, Chen Y, Ma Q, Fang J, He J, Cheng YQ, et al. Effect of betulinic acid on the regulation of Hiwi and cyclin B1 in human gastric adenocarcinoma AGS cells. Acta Pharmacol Sin 2010; 31: 66-72.

18 Cheng YQ, Chen Y, Wu QL, Fang J, Yang $\sqcup$. Effect of betulinic acid on inducing apoptosis of human multiple myeloma cell line RPMI-8226. Zhongguo Shi Yan Xue Ye Xue Za Zhi 2009; 17: 1224-9. chinese.

19 Pandey MK, Sung B, Aggarwal BB. Betulinic acid suppresses STAT3 activation pathway through induction of protein tyrosine phosphatase SHP-1 in human multiple myeloma cells. Int J Cancer 2010; 127: 282-92.

20 Fulda S, Kroemer G. Targeting mitochondrial apoptosis by betulinic acid in human cancers. Drug Discov Today 2009; 14: 885-90.

21 Mullauer FB, Kessler JH, Medema JP. Betulinic acid, a natural compound with potent anticancer effects. Anticancer Drugs 2010; 21: 215-27.

22 Mizushima N, Yoshimori T, Levine B. Methods in mammalian autophagy research. Cell 2010; 140: 313-26.

23 Luo S, Rubinsztein DC. Apoptosis blocks Beclin 1-dependent autophagosome synthesis: an effect rescued by Bcl-xL. Cell Death Differ 2010; 17: 268-77.

24 Boya P, Gonzalez-Polo RA, Casares N, Perfettini JL, Dessen P, Larochette $\mathrm{N}$, et al. Inhibition of macroautophagy triggers apoptosis. Mol Cell Biol 2005; 25: 1025-40.

25 Selzer E, Pimentel E, Wacheck V, Schlegel W, Pehamberger H, Jansen $B$, et al. Effects of betulinic acid alone and in combination with irradiation in human melanoma cells. J Invest Dermatol 2000; 114: 935-40.

26 Zuco V, Supino R, Righetti SC, Cleris L, Marchesi E, Gambacorti-Passerini $\mathrm{C}$, et al. Selective cytotoxicity of betulinic acid on tumor cell lines, but not on normal cells. Cancer Lett 2002; 175: 17-25.

27 Fulda S, Friesen C, Los M, Scaffidi C, Mier W, Benedict M, et al. Betulinic acid triggers CD95 (APO-1/Fas)- and p53-independent apoptosis via activation of caspases in neuroectodermal tumors. Cancer Res 1997; 57: 4956-64.

28 Lazebnik YA, Kaufmann SH, Desnoyers S, Poirier GG, Earnshaw WC. Cleavage of poly(ADP-ribose) polymerase by a proteinase with properties like ICE. Nature 1994; 371: 346-7.

29 Klionsky DJ, Abeliovich H, Agostinis P, Agrawal DK, Aliev G, Askew DS, et al. Guidelines for the use and interpretation of assays for monitoring autophagy in higher eukaryotes. Autophagy 2008; 4: 151-75.

30 Liu L, Yang M, Kang R, Wang Z, Zhao Y, Yu Y, et al. DAMP-mediated autophagy contributes to drug resistance. Autophagy 2011; 7: 112-4.

31 Chen S, Rehman SK, Zhang W, Wen A, Yao L, Zhang J. Autophagy is a therapeutic target in anticancer drug resistance. Biochim Biophys Acta 2010; 1806: 220-9. 